Pacific Journal of Mathematics

INVARIANT SUBMANIFOLDS OF FREE CYCLIC ACTIONS ON 


\section{INVARIANT SUBMANIFOLDS OF FREE CYCLIC ACTIONS ON SPHERES}

\section{SUSAN SZCZEPANSKI}

Let $\mathrm{Z}_{m}$ be the cyclic group of order $m$. Denote by $\rho$ a free PL (orientation preserving) action of $\mathbf{Z}_{m}$ on the sphere $S^{2 k+1}, k \geq 3$. In this paper, we study submanifolds, $K^{2 k-1}$, of the sphere which are left invariant by the free action $\rho$. In particular, the submanifolds considered are highly conneced, that is, $\pi_{1}(K)=0, i<k-1$. We apply the techniques of b-surgery theory as developed in [Szczepanski, 1983] and some new results in this homology surgery theory which we develop herein. We obtain a classification (up to cobordism) of invariant submanifolds with given restriction $\tau=\rho \mid K$ and a relationship between invariant highly connected submanifolds and invariant (homotopy) spheres.

Introduction. Let $\rho$ denote a free PL (orientation preserving) action of $\mathbf{Z}_{m}$, the cyclic group of order $m$, on the sphere $S^{2 k+1}$. Two questions involving submanifolds left invariant under such an action come to mind. The first involves the determination of which $\mathbf{Z}_{m}$ actions on which manifolds of dimension $2 k-1$ can arise as the restriction of $\rho$ to an invariant submanifold. The second is a question of uniqueness or classification of equivariant embeddings. That is, if it is known that a given submanifold $K^{2 k-1}$ of the sphere $S^{2 k+1}$ has a free PL (orientation preserving) action of $\mathbf{Z}_{m}$, denoted by $\tau$, which is the restriction of $\rho$, it is reasonable to ask if this equivariant embedding of the pair $(K, \tau)$ is unique (up to an appropriate notion of equivalence) and to seek a classification of all such equivariant embeddings of $(K, \tau)$ in the case of non-uniqueness. The case of invariant subspheres was addressed in the work of Cappell and Shaneson using homology equivalences and homology surgery obstruction groups (see [CS, 1974], §9-10), and in the work of Stolzfus by using Seifert matrices (see [St, 1977], §6). Here we will be concerned with the classification problem of equivariant embeddings of more general submanifolds up to the equivalence relation known as equivariant cobordism (see $\S 1$ ). In particular we are interested in submanifolds of spheres which are highly connected. These are manifolds for which the homotopy groups vanish below the middle dimension, $\pi_{i}(K)=$ $0, i<k-1$. This class of submanifolds of the sphere arises naturally in the study of isolated singularities of complex hypersurfaces. 
Our methods follow the philosophical approach of Cappell and Shaneson and involve a new homology surgery theory, $\mathbf{b}$-surgery theory as developed in [Sz, 1983; §1]. The results include a classification (up to equivariant cobordism) of invariant highly connected submanifolds with a given free $\mathbf{Z}_{m}$ action and the establishment of a relationship between the highly connected case and the spherical case.

Let $\tau=\rho \mid \iota(K)$ denote the restriction of $\rho$ to the highly connected submanifold, $\iota(K)$, where $\iota$ is used to denote an embedding of $K$ into the sphere $S^{2 k+1}$. The main geometric results are summarized in the following.

TheOREM A (See Thms. 3.1, 4.5, 4.7). The group of cobordism classes of invariant (homotopy) spheres $\iota_{0}: \Sigma \subset S^{2 k+1}$ with $\rho \mid \iota_{0}\left(\Sigma_{0}\right)=\tau_{0}$ acts on the set of cobordism classes of invariant submanifolds $\iota$ : $K \subset S^{2 k+1}$ with $\rho \mid \iota(K)=\tau$.

(i) If $H^{k-1}(K) \neq 0$, this action is not transitive; the orbit space contains an infinite set;

(ii) If $H^{k-1}(K)=0$ and either $k$ or $m$ is odd, then this action is transitive;

(iii) If $H^{k-1}(K)=0$ and both $k$ and $m$ are even, the orbit space of the action has at most two elements.

Thus, we see that the variety of ways in which a manifold $K$ with free $\mathbf{Z}_{m}$ action $\tau$ may arise as the restriction of $\rho$ is related to the variety of ways in which a homotopy sphere with free $\mathbf{Z}_{m}$ action $\tau_{0}$ so arises. There is an infinitely greater variety of invariant submanifolds $(K, \tau)$ whenever $H^{k-1}(K) \neq 0$. If $k$ or $m$ is odd, we have a greater variety only if $H^{k-1}(K) \neq 0$. If $k$ and $m$ are both even the variety is greater by at most a factor of two whenever $H^{k-1}(K)=0$.

For submanifolds of $S^{2 k+1}$ PL homeomorphic to the sphere $S^{2 k-1}$, the questions of which actions can arise as restrictions of an action on $S^{2 k+1}$, and in how many distinct ways can a given action on $S^{2 k+1}$ so arise are answered in [CS, 1974]. We are interested in studying highly connected submanifolds, that is, submanifolds $K^{2 k-1} \subset S^{2 k+1}$ with $\pi_{i}(K)=0, i<k-1$, invariant under the action $\rho$. Theorem A follows in part from our classification of $C(\rho, \tau)$, the set of equivariant cobordism classes of invariant submanifolds $K \subset S^{2 k+1}$ for which the restriction of $\rho$ is equivalent to the given action of $\tau$ on $K$ (see $\S 1$ ).

Let $\mathscr{F}: \mathbf{Z}[\mathbf{Z}] \rightarrow \mathbf{Z}\left[\mathbf{Z}_{m}\right]$, and $A=\pi_{k-1}(K)$. The philosophical approach found in $[$ CS, $1974 ; \S 10]$ requires us to study maps of pairs $h:(V, \partial V) \rightarrow(X, Y)$. In the case of invariant subspheres, it is valid to 
assume the restriction $h \mid \partial V$ is a homology equivalence over the coefficients $\mathbf{Z}\left[\mathbf{Z}_{m}\right]$. To obtain results, surgery is performed relative $h \mid \partial V$ in the hope of obtaining a homology equivalence over $\mathbf{Z}\left[\mathbf{Z}_{m}\right]$ to replace the map $h$. There is an obstruction to performing these surgeries successfully; it is an element of the surgery group $\Gamma_{2 k+2}(\mathscr{F})$. When the philosophical approach is applied to the more general problem of invariant highly connected submanifolds, again we wish to study maps of pairs $h:(V, \partial V)$ $\rightarrow(X, Y)$ but in this case it is not true that the restriction $h \mid \partial V$, is a homology equivalence. For this problem, surgery relative $h \mid \partial V$ is performed with the hope of producing a replacement for $h$ which satisfies certain homological prerequisites. In particular, there is no hope of doing surgery relative $h \mid \partial V$ and producing a homology equivalence. Even if we could, it would not lead to the results for which we hope. The surgery will be successful if it produces a map $\tilde{h}:(\tilde{V}, \partial V) \rightarrow(X, Y)$ which homologically (over $\mathbf{Z}\left[\mathbf{Z}_{m}\right]$ ) is no more complicated than what is dictated by $h \mid \partial V$ (see [sz 1983; §1] for details). The obstruction to replacing $h$ by a suitable map is an element of the $\mathrm{b}$-surgery group $\mathrm{b}_{2 k+2}(\mathscr{F} ; A \oplus A)$.

Let $\tilde{B}$ denote the orbit space of the action on an invariant subgroup $B \subset \mathbf{b}_{2 k+2}(\mathscr{F} ; A \oplus A)$ induced by the action of the Wall group $L_{2 k+2}(\mathbf{Z})$ on $\mathbf{b}_{2 k+2}(\mathscr{F} ; A \oplus A)$, (see [Sz 1983; §2]). Our classification takes the following form.

Theorem B (See Thms. 1.5, 2.2, 3.3). There exists a subgroup $B \subset$ $\mathbf{b}_{2 k+2}(\mathscr{F} ; A \oplus A)$ such that $\tilde{B}$ acts freely on $C(\rho, \tau)$ with orbit space

$$
O(\tau)= \begin{cases}0, & k \text { odd }, \text { m even } \\ \mathbf{Z}_{2}, & \text { k odd }, \text { m odd } \\ \mathbf{Z}, & \text { k even } .\end{cases}
$$

The subgroup $B$ is given explicitly in terms of normal invariants and a pairing of the groups $\mathrm{5}_{2 k+2}(\mathscr{F} ; A \oplus A)$ and $\Gamma_{2 k+2}(\mathscr{F})$ with values in $L_{2 k+2}\left(\mathbf{Z}_{m}\right)$.

Let $\tau_{0}$ be a free $\mathbf{Z}_{m}$ action on $S^{2 k+1}$ arising as the restriction of $\rho$ to an invariant sphere. The relationship between the sets of cobordism classes $C\left(\rho, \tau_{0}\right)$ and $C(\rho, \tau)$ corresponds to the relationship between the homology surgery groups $\Gamma_{2 k+2}(\mathscr{F})$ and $\mathbf{b}_{2 k+2}(\mathscr{F} ; A \oplus A)$. That is, there is a geometrically defined operation, tunnel sum (see [CS, 1974]; §9), which gives a group structure for $C\left(\rho, \tau_{0}\right)$ and a group action of $C\left(\rho, \tau_{0}\right)$ on $C(\rho, \tau)$. There is an action of $\Gamma_{2 k+2}(\mathscr{F})$ on $\mathbf{b}_{2 k+2}(\mathscr{F} ; A \oplus A)$ which is given algebraically by taking orthogonal direct sum of forms. The 
operation of tunnel sum can be described algebraically by this action. Let $B_{0}$ and $B$ be the subgroups of Theorem B for $C\left(\rho, \tau_{0}\right)$ and $C(\rho, \tau)$ respectively.

THeOREM C (See Thms. 3.1 and 3.2). The algebraic action of $\Gamma_{2 k+2}(\mathscr{F})$ on $\mathbf{5}_{2 k+2}(\mathscr{F} ; A \oplus A)$ induces an action of $\tilde{B}_{0}$ on $\tilde{B}$. This induced action corresponds to the action of tunnel sum, that is,

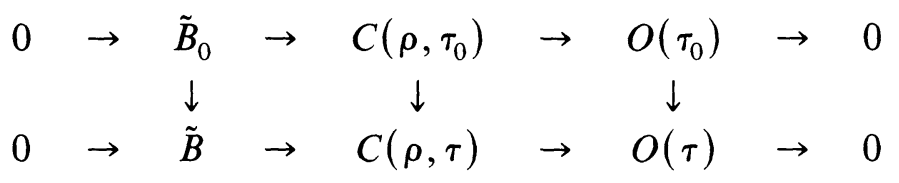

is a commutative diagram.

We then reduce the geometric problem of Theorem A to an algebraic one by the following.

Theorem D (See Thms. 3.1 and 3.2). The orbit spaces of the two actions are isomorphic, that is,

$$
\mathbf{b}_{2 k+2}(\mathscr{F} ; A \oplus A) / \Gamma_{2 k+2}(\mathscr{F}) \cong C(\rho, \tau) / C\left(\rho, \tau_{0}\right) .
$$

The statements (ii) and (iii) of Theorem A follow from a reciprocity principle for 6-surgery theory [Sz 2].

ThEOREM E (See Thm. 4.5). If $A^{*}=\operatorname{Hom}(A, \Lambda)=0$, where $\mathscr{F}: \mathbf{Z}[\pi] \rightarrow \Lambda$, then

$$
\mathbf{b}_{2 k+2}(\mathscr{F} ; A \oplus A) / \Gamma_{2 k+2}(\mathscr{F}) \cong \Gamma_{2 k+3}(\mathscr{F}) / \mathbf{b}_{2 k+3}(\mathscr{F} ; A \oplus A) .
$$

In the case $A^{*} \neq 0$ and $\Lambda$ is the integral group ring of a finite group, we show that the orbit space of the algebraic action is not finite (see $\$ 4$ ); hence we may conclude that the reciprocity formula of Theorem $\mathrm{E}$ does not hold if $A^{*} \neq 0$, in the case $\Lambda=\mathbf{Z}\left[\mathbf{Z}_{m}\right]$, and we obtain statement (i) of Theorem A.

1. Let $\mathbf{Z}_{m}$ be the cyclic group of order $m$. Given a free orientation preserving PL action of $\mathbf{Z}_{m}$ on a PL manifold, $\tilde{M}$, the quotient space of the action, $M$, is an orientated PL manifold with an identification of its fundamental group with the group $\mathbf{Z}_{m}$. Two actions on a manifold will be called equivalent (homotopy equivalent) if their quotient spaces are PL homeomorphic (homotopy equivalent) by a degree one map that preserves the identifications of the fundamental groups with $\mathbf{Z}_{m}$. 
As in [CS, 197.4], we define the suspension of a free action to be any free action obtained from a given one by taking its join with a free action on $S^{1}$; the quotient manifold of a suspension will be called the suspension of the quotient manifold. There is one suspension for each primitive $m$ th root of unity. Recall from [CS] that the quotient space of an action of $\mathbf{Z}_{m}$ on a sphere is called a fake lens space, and the different suspensions of a given fake lens space give rise to different actions on $S^{2 k+1}$, that is, actions which are not homotopically equivalent. If $L^{2 k-1} \subset M^{2 k+1}$ is an inclusion of fake lens spaces, the normal bundle of $L$ in $M$ is a 2-disk bundle with Chern class a generator of $H^{2}(L ; \mathbf{Z})=\mathbf{Z}_{m}$; this generator determines a suspension, $N$, of the fake lens space $L$.

Let $\rho$ be a free action of $\mathbf{Z}_{m}$ on $S^{2 k+1}$ with $M=S^{2 k+1} / \rho$. In this paper, we are interested in invariant codimension two submanifolds which are highly connected, that is, submanifolds $K^{2 k-1} \subset S^{2 k+1}$ invariant under the action of $\rho$ and satisfying $\pi_{l}(K)=0$ for $i<k-1$. Let $J$ be the quotient space of the restriction of $\rho$ to $K$. Assume $k \geq 3$.

We begin by introducing the notion of the characteristic map for the restriction $\rho \mid K$. This important tool provides a linear lens space against which we "measure" the invariant highly connected submanifold $K$.

According to [Wall, Ch. 14], given a primitive $m$ th root of unity, every homotopy lens space is homotopy equivalent to the corresponding suspension of a unique linear lens space of the form $L=L(m ; 1,1, \ldots, 1, c)$ with $(c, m)=1$. If $M$ contains a manifold $J$ as described above, we will construct the homotopy equivalence to be an extension of a map from $J$ to the linear lens space $L$. We call this homotopy equivalence the characteristic map for the restriction $\rho \mid K$.

Proposition 1.1. There exists a suspension $N$ of a linear lens space $L$ and a homotopy equivalence $f: M \rightarrow N$ such that $f^{-1}(L)=J$ and $f \mid J$ : $J \rightarrow L$ is a highly connected degree one map. That is, the map induced by $f \mid J$ on homotopy group $(f \mid J)_{*}: \pi_{i}(J) \rightarrow \pi_{i}(L)$ is an isomorphism for $i<k-1$. The map $f$ is unique up to a homotopy relative a map of bundle neighborhoods $f \mid R(J): R(J) \rightarrow R(L)$.

The proof of the proposition relies on a well known argument used to establish [CS, Prop. 6.3] in the case of a sphere, and [Sz, Theorem 2.10] in the highly connected case. We include it here for the sake of the reader.

LEMMA 1.2. An embedding of a highly connected codimension two manifold $K$ into a sphere $S^{2 k+1}$ can be factored through an embedding of $K$ into a bundle neighborhood of the standard embedding of $S^{2 k-1}$. The 
inclusion of $K$ into $S^{2 k-1} \times D^{2}$ followed by the projection unto the zero section is a degree one highly connected map from $K$ to $S^{2 k-1}$.

Proof. Let $S^{1} \rightarrow S^{2 k+1}-K$ be a generator of $H_{1}\left(S^{2 k+1}-K\right) \cong \mathbf{Z}$ corresponding to the canonical generator of the first homology of the circle bundle over $K$ determined by the embedding of $K$. Because of the large codimension, this copy of $S^{1}$ is unique up to isotopy. The embedding of $K$ clearly is contained in the complement of the interior of a neighborhood $S^{1} \times D^{2 k}$; so $K \subset S^{2 k-1} \times D^{2} \subset S^{2 k+1}$. To see that the inclusion of $K$ into this $S^{2 k-1} \times D^{2}$ followed by the bundle projection is a degree one map, we consider $W=S^{2 k-1} \times D^{2}-\operatorname{int}\left(K \times D^{2}\right)$. In the case $K$ is a sphere, $W$ is a homolgoy $h$-cobordism. In the case $K$ is just highly connected, the inclusions of the boundary components will fail to induce an isomorphism of homology groups in dimensions $k-1$ and $k$. In both cases however, the inclusions do induce an isomorphism in dimension $2 k-1$. It follows from a Mayer-Vietoris sequence that the map from $K$ to $S^{2 k-1}$ described above is degree one.

Proof of Proposition 1.1. By taking advantage of the cyclic action, we may use Lemma 1.2 to factor the embedding $J \subset M$ through an embedding into a submanifold of $M$ homotopy equivalent to a bundle over a lens space. As above, we use transversality to represent a generator of $\pi_{1}(M)$ by a circle in $(M-J)$ and obtain a decomposition of $M$ as the union along the boundary of $S^{1} \times D^{2 k}$ and a $(2 k+1)$-manifold $P$ with $J \subset$ int $P$. We may lift this decomposition to the sphere $S^{2 k+1}$ and obtain the factorization as in Lemma 1.2 for the invariant submanifold $K$. Thus, $P$ is the quotient space of the restriction of $\rho$ to $S^{2 k-1} \times D^{2}$.

Now, $M$ is homotopy equivalent to a linear lens space of dimension $2 k+1$ and the homotopy equivalence can be split along a circle representing a generator, as done above. Hence we see that $P$ is homotopy equivalent to a bundle over a linear lens space $L$. Thus, the equivariant embedding of $K$ into $S^{2 k+1}$ can be factored through an embedding into a bundle over a sphere, and the embedding of $J$ in the fake lens space $M$ can be factored through an embedding into a space homotopy equivalent to a bundle over a linear lens space. The restriction of the homotopy equivalence followed by the bundle projection is certainly highly connected. To see that it is a degree one map, it suffices to establish this for the map of the universal covering spaces from $K$ to $S^{2 k-1}$ which covers this map. But this follows from Lemma 1.2. 
Now let $N$ be the suspension of $L$ determined by $E(L)$, the normal bundle of $L$ in the $(2 k+1)$-linear lens space. We begin with the degree one map $J \rightarrow L$, extend this to a homotopy equivalence from $P$ to $E(L)$ and then extend this to a map $f: M \rightarrow N$ which has all of the desired properties.

If $P$ were actually a bundle over the lens space $L$, we could proceed as in [Sz, Lemma 2.2] and produce the characteristic map of the semi-local knot $J \subset P$. For the more general case at hand, it is still true that the obstructions all lie in cohomology groups which must vanish since $P$ is homotopy equivalent to such a bundle. It is fairly obvious that once defined on $\partial P$, there is no obstruction to extending this to a map $f: M \rightarrow N$. That $f$ is a homotopy equivalence and the extent to which it is unique follows from [Sz, Lemmas 2.2 and 2.3].

Related to the notion of characteristic map for the restriction $\rho \mid K$ is the notion of highly connected characteristic submanifolds. A highly connected characteristic map of submanifolds associated to a homotopy equivalence $f: M \rightarrow N$, where $N$ is a suspension of $L^{2 k-1}$, is a restriction map $g \mid g^{-1} L: g^{-1} L \rightarrow L$ where $g: M \rightarrow N$ is homotopic to $f$, transverse to $L$ and $g \mid g^{-1} L$ is a highly connected map. If $g \mid g^{-1} L$ is actually a homotopy equivalence, $g^{-1} L$ is called a characteristic submanifold. In what follows, we will use the phrase "highly connected characteristic submanifold" to describe the submanifold $g^{-1} L$ together with the restriction map, $g \mid g^{-1} L$.

Reviewing [CS], we note that in the case of an invariant sphere of $\rho$, $L$ may be chosen to be the quotient space of the restriction of $\rho$. The characteristic map then is an extension of the identity map on $L[\mathbf{C S}, \mathrm{p}$. 325]. Further, $P$ is a bundle over a lens space and the embedding $L \subset M$ factors through a semi-local knot $L \subset E(L)=P$ (see [CS, p. 302]). Thus it is shown [CS, Prop. 9.1] that characteristic submanifolds of $M$ are exactly the quotient spaces of invariant spheres of $\rho$. In like manner, it follows from our definitions and Proposition 1.1 that an analogous statement can be made in the highly connected case where $P$ is homotopy equivalent to $E(L)$. If in addition to $J$, the homotopy lens space $M$ contains a characteristic submanifold, i.e. a codimension two homotopy lens space, $L$, then $P$ is in fact a bundle over $L$ and the embedding $J \subset M$ factors through a semi-local knot $J \subset E(L)=P$ over a highly connected degree one map $g \mid J: J \rightarrow L$ (see [Sz, p. 29]). In [CS, 9.2], it is stated that the obstruction to the existence of $L$ lies in $\mathbf{Z}_{2}$ and is zero if $k$ and $m$ are not both even. This discussion allows us to make the following statement. 
Proposition 1.3. The quotient spaces of highly connected invariant submanifolds of $\rho$ are exactly the highly connected characteristic submanifolds of $M=S^{2 k+1} / \rho$.

Our first goal is to classify up to cobordism all invariant embeddings of $K$ into $S^{2 k+1}$. Let $K_{0}$ and $K_{1}$ be two such invariant embeddings. There is a $\mathbf{Z}_{m}$ action, $\bar{\rho}$, on $S^{2 k+1} \times I$ obtained by taking the action of $\rho$ on the first factor and the trivial action on the second. We say that $K_{0}$ and $K_{1}$ are equivariantly cobordant if there exists a submanifold $W$ of $S^{2 k+1} \times I$, invariant under the action $\bar{\rho}$, PL equivariantly homeomorphic to $K \times I$ and meeting the boundary transversely in $\partial W=\left(K_{0} \times 0\right) \cup$ $\left(K_{1} \times 1\right)$. Note that in particular, $\rho \mid K_{0}$ and $\rho \mid K_{1}$ are equivalent.

Let $\tau$ denote a $\mathbf{Z}_{m}$ action on a highly connected manifold $K^{2 k-1}$. Suppose that $\rho$ has an invariant submanifold equivalent to $(K, \tau)$. The set of equivariant cobordism classes of invariant submanifolds for which the restriction of $\rho$ is equivalent to $\tau$ is denoted by $C(\rho, \tau)$.

We now use the tools set up in Propositions 1.1 and 1.3 to see that there is a suspension $N$ of a linear lens space $L$ and a unique homotopy equivalence $f: M \rightarrow N$ preserving polarization (i.e., orientation and identification of fundamental groups) for which $J=K / \tau$ is a highly connected characteristic submanifold. Two such submanifolds associated to $f$, say $J_{i}=g_{i}^{-1} L, i=0,1$, are cobordant if and only if there exists a homotopy $G$ of the maps $g_{0}$ and $g_{1}$ transverse regular to $L \times I$ with $G \mid G^{-1}(L \times I)$ a map of $s$-cobordisms into $L \times I$.

An argument similar to the discussion leading up to Proposition 1.1 shows that the classes $C(\rho, \tau)$ correspond exactly to the cobordism classes of highly connected characteristic submanifolds for $f$ that are PL homeomorphic to $J$ via a polarization preserving map. Having identified the appropriate description of the objects we wish to study, we now proceed and look for a classification in terms of certain surgery obstruction groups. We first verify that certain homological conditions are satisfied and that all is set up so that an element in such an obstruction group can be defined.

Let $W=\mathrm{Cl}(M-R(J))$ be the closed complement of a bundle neighborhood of $J$ such that the restriction $f \mid W$ is a map to $D^{2 k} \times S^{1}=$ $\mathrm{Cl}(N-R(L))$. Let $\mathscr{F}: \mathbf{Z}[\mathbf{Z}] \rightarrow \mathbf{Z}\left[\mathbf{Z}_{m}\right]$ be the map induced by the map of fundamental groups which in turn is induced by the inclusion $D^{2 k} \times S^{1}$ $\subset N$. As a consequence of Proposition 1.1 and Lemma 1.2, we observe that $f \mid W$ is a degree one normal map and the induced map on homology with local coefficient, $\Lambda=\mathbf{Z}\left[\mathbf{Z}_{m}\right]$, enables us to conclude that $f \mid W$ is 
$\Lambda$-homology highly connected, that is, the map induced by $f \mid W$ on the homology groups with coefficients in $\Lambda$ is an isomorphism in dimension $i \leq k-1$. In other words, $K_{i}(W ; \Lambda)=0$ for $i \leq k-1$, where $K_{i}(W ; \Lambda)$ denotes the kernel of the split surjections of homology groups induced by $f \mid W$, (see [Wall, 2.2] and related discussion for more detailed description of these kernel groups).

LEMMA 1.4. Let $f: M \rightarrow N$ be the characteristic map for $J \subset M$. Then, for all $j$, the sequences

$$
0 \rightarrow K_{j+1}(W, \partial R ; \Lambda) \rightarrow K_{j}(\partial W ; \Lambda) \rightarrow K_{j}(W ; \Lambda) \rightarrow 0
$$

and

$$
0 \rightarrow K_{j+1}(R(J), \partial R ; \Lambda) \rightarrow K_{j}(\partial R ; \Lambda) \rightarrow K_{j}(R(J) ; \Lambda) \rightarrow 0
$$

are exact, and

$$
K_{j}(\partial R ; \Lambda)=K_{j}(R(J) ; \Lambda) \oplus K_{j}(W ; \Lambda) .
$$

Proof. This follows immediately from Proposition 1.1 and the MayerVietoris sequence.

Let $A=K_{k-1}(J ; \Lambda)$. It follows from the above and the fact that $f \mid J$ is highly connected that $K_{k-1}(\partial R ; \Lambda)$ is isomorphic to $A$ as a $\Lambda$-module, and that $K_{i}(\partial R ; \Lambda)=0$ for $i \leq k-2$. Also, $K_{k}(W ; \Lambda)$ is isomorphic to $A$ (as a $\Lambda$-module); this follows in part from the high connectivity and from duality.

Let $f_{0}$ and $f_{1}$ be characteristic maps for $J_{0}$ and $J_{1}$, respectively, where $J_{0}$ and $J_{1}$ represent elements of $C(\rho, \tau)$. Suppose that their restrictions to the respective complements $W_{0}$ and $W_{1}$ are normally cobordant relative $\partial R$ with underlying manifold $Z$. From the Mayer-Vietoris sequence for $\partial Z=W_{0} \cup W_{1}$, we obtain an identification

$$
A \oplus A=K_{k}(\partial R ; \Lambda) \stackrel{\pi_{1}}{\rightarrow} K_{k}\left(W_{i} ; \Lambda\right)=A
$$

where $\pi_{1}$ is the projection map [Sz, p. 36]. This cobordism determines an element in the surgery obstruction group, $\gamma \in \mathbf{b}_{2 k+2}(\mathscr{F} ; A \oplus A)$. This element, $\gamma$, is the obstruction to performing surgery on the interior of $Z$ which results in a new manifold, $\tilde{Z}$, which homologically (over $\Lambda$ ) is an $h$-cobordism. As the cobordism is relative $\partial R$, we obtain a normal cobordism $(F, B):(Z \cup R(J) \times I) \rightarrow N \times I$ between $f_{0}$ and $f_{1}$ by taking the union with $f \mid R(J) \times \mathrm{id}_{I}$ along $\partial R \times I$. (Recall that $J_{0}$ and $J_{1}$ 
are highly connected characteristic submanifolds for a homotopy equivalence $f: M \rightarrow N$.) This process of "glueing in" describes geometrically an algebraically defined homomorphism

$$
1_{*}: \mathbf{b}_{2 k+2}(\mathscr{F} ; A \oplus A) \rightarrow L_{2 k+2}\left(\mathbf{Z}_{m}\right)
$$

with $1_{*}(\gamma)=\sigma(F, B)$. The algebraic definition of this homomorphism will be given in §3; briefly, it is the homomorphism obtained from a pairing of $\mathbf{b}_{2 k+2}(\mathscr{F} ; A \oplus A)$ and $\mathbf{b}_{2 k+2}\left(\mathrm{id}_{\mathbf{z}_{m}} ; A \oplus A\right)$ with values in $L_{2 k+2}\left(\mathbf{Z}_{m}\right)$ by considering the "orbit" of the trivial element of $\mathbf{b}_{2 k+2}\left(\mathrm{id}_{\mathbf{z}_{m}} ; A \oplus A\right)$. Using the definition of the $\mathbf{b}$-surgery group and the notation in [Sz, §1], we may describe it as a "forgetful" homomorphism; if $(\lambda: H \rightarrow G$; $(G ; \theta, \psi))$ represents $\gamma \in \mathbf{b}_{2 k+2}(\mathscr{F} ; A \oplus A)$, then $j_{*}(\gamma)$ may be represented by the unimodular form obtained from $(G ; \theta, \psi)$ by tensoring with $\mathbf{Z}_{m}$.

From the above discussion and the fact that the process of glueing in results in a cobordism from $N$ to itself, we conclude that the image in $L_{2 k+2}\left(\mathbf{Z}_{m}\right)$ of an element of $\mathbf{b}_{2 k+2}(\mathscr{F} ; A \oplus A)$ arising in the geometric setting currently under study lies in the image of the surgery obstruction map

$$
s:[\Sigma N, G / \mathrm{PL}] \rightarrow L_{2 k+2}\left(\mathbf{Z}_{m}\right) .
$$

Let $B \subseteq \mathbf{b}_{2 k+2}(\mathscr{F} ; A \oplus A)$ be the subgroup consisting of all elements with this property. By the realization theorem of $\mathbf{b}$-surgery theory $[\mathbf{S z}$, Theorem 1.11], each $\gamma \in \mathrm{b}_{2 k+2}(\mathscr{F} ; A \oplus A)$ determines a map $(H, C)$ which is a normal cobordism, relative $\partial R$, of $f_{0} \mid W_{0}$ to a map $h: Y \rightarrow D^{2 k} \times S^{1}$ for which the conclusion of Lemma 1.4 holds and for which $\sigma(H, C)=\gamma$. The image of $B$ in $L_{2 k+2}\left(\mathbf{Z}_{m}\right)$ acts trivially on the homotopy classes of maps into $N$; hence if $\gamma \in B$, then $h \cup f \mid R(J)$ is a characteristic map for a representative of an element of $C(\rho, \tau)$.

Besides the algebraic action described above, there is a well-defined action of $L_{2 k+2}^{h}(\mathbf{Z})$ on $\mathbf{b}_{2 k+2}(\mathscr{F} ; A \oplus A)$ which is given by orthogonal direct sum of forms [Sz, p. 42]. In this setting, this action may be realized geometrically by taking connected sum with a map to $S^{2 k+2}$ with surgery obstruction in $L_{2 k+2}(e) \cong L_{2 k+2}(\mathbf{Z})$. We are now prepared to describe a relationship between the geometric $C(\rho, \tau)$ and the algebraic $\mathrm{b}_{2 k+2}(\mathscr{F} ; A \oplus A)$.

THEOREM 1.5. The subgroup $B \subseteq \mathbf{b}_{2 k+2}(\mathscr{F} ; A \oplus A)$ acts on the set $C(\rho, \tau)$. Moreover, there is an action on $B$ induced by the action of $L_{2 k+2}(\mathbf{Z})$ on $\mathbf{b}_{2 k+2}(\mathscr{F} ; A \oplus A)$; the orbit space of this action, $\tilde{B}$ acts freely on $C(\rho, \tau)$. 
Proof. That the action on $C(\rho, \tau)$ described in the above discussion is well-defined follows by an argument similar to that in [Sz, Theorem 2.7], applied to the restriction of $f$ to the manifold $P$ obtained in the proof of Proposition 1.1.

The second statement requires a description of the elements of $B$ which act trivially.

Let $D \subseteq L_{2 k+2}(\mathbf{Z})$ be the image of the surgery obstruction map

$$
s:\left[\Sigma^{2} S^{2 k-1} \times S^{1}, G / \mathrm{PL}\right] \rightarrow L_{2 k+2}(\mathbf{Z}) .
$$

First, by restricting the action of $L_{2 k+2}(\mathbf{Z})$ to the subgroup $D$, we obtain an action of $D$ on $B \subseteq \mathrm{b}_{2 k+2}(\mathscr{F} ; A \oplus A)$. Let $(H, C): Z \rightarrow D^{2 k} \times S^{1}$ $\times I$ be a normal cobordism, relative $\partial R \times I$, from $f_{0}$ to $f_{1}$ with $\sigma(H, C)$ $=\gamma \in B$. We realize $\delta$ by performing surgeries in a neighborhood of the boundary component $\partial R \times I$. Let $(G, B)$ be the normal cobordism from $H \mid \partial R \times I$ to itself, relative $(\partial R \times 0) \cup(\partial R \times 1)$ obtained from

$$
H \mid \partial R \times \text { Id } \times \text { Id; } \partial R \times I \times I \rightarrow\left(S^{2 k-1} \times S^{1}\right) \times I \times I
$$

by taking connected sum with a normal map to $S^{2 k+2}$ which represents $\delta \in L_{2 k+2}(e) \cong L_{2 k+2}(\mathbf{Z})$. Taking the union along $\partial R \times 0 \times I$, the algebraic action of $D$ on $B$ given by orthogonal sum of forms is realized geometrically, that is, $\sigma(G \cup H)=\delta \cdot \gamma$.

It remains to show that if $\gamma \in B$ acts trivially, then $\gamma$ is in the orbit of the zero element of $\mathbf{b}_{2 k+2}(\mathscr{F} ; A \oplus A)$; that is, there exists a $\delta \in D$ such that $\delta \cdot 0=\gamma$. If $\gamma$ acts trivially, $(H, C)$ is a cobordism from $f_{0}$ to itself. Let $F=f_{0} \times \mathrm{id}$; this represents the zero element. Consider the map $H \cup F$, the union taken along $H\left|\partial R \times I=f_{0}\right| \partial R \times$ id. This gives a cobordism from $f_{0} \cup-f_{0}$ to itself; we keep in mind that this is normally cobordant to the identity on $S^{2 k} \times S^{1}$. Now consider the surgery obstruction of $G$ into $S^{2 k} \times S^{1} \times S^{1}$ obtained from $H \cup F$ by identifying along the map $f_{0} \cup f_{0}$. By the Kunneth formula of [Sh, 5.1], $L_{2 k+2}(\mathbf{Z} \oplus \mathbf{Z}) \cong$ $L_{2 k+1}(\mathbf{Z}) \oplus L_{2 k+2}(\mathbf{Z})$ and so $\sigma(G)$ is determined by $\sigma\left(f_{0} \cup-f_{0}\right)=0$ and $\sigma \in L_{2 k+2}(\mathbf{Z})$, where $\sigma$ is the surgery obstruction associated to the map $H \cup F$. Let $H_{0}$ be a normal map from $f_{0} \mid \partial R \times$ id to itself with obstruction - $\sigma$. Applying the constructions and analysis of $G$ as above to the map $H_{0} \cup H \cup F$, we find that the element in $L_{2 k+2}(\mathrm{Z})$ vanishes. It follows that $\sigma\left(H_{0} \cup H\right) \in \mathrm{b}_{2 k+2}(\mathscr{F} ; A \oplus A)$ vanishes and so, taking $\sigma=\delta$, we obtain $-\delta \cdot \gamma=0$, or that $\gamma$ is in the orbit of the trivial element.

Thus, having defined $C(\rho, \tau)$, we are able gather a fair amount of information concerning its structure by applying notions from surgery theory. In particular, the realization of surgery obstructions by cobor- 
disms and the various relationships one can establish algebraically and sometimes geometrically among different surgery theories and surgery groups played an important role in our discussions so far. We turn now to an investigation of some of the underlying algebra.

2. In this section, we define and describe a homomorphism $1_{*}: \mathbf{b}_{2 n}(\mathscr{F} ; A \oplus B) \rightarrow L_{2 n}(\Lambda)$ where $\mathscr{F}: \mathbf{Z}[\pi] \rightarrow \Lambda$ is a ring epimorphism. In the preceding section, we indicated how this homomorphism enters the study of invariant highly connected submanifolds of a free cyclic action on a sphere. In particular, we saw that

$$
1_{*}: \mathbf{b}_{2 k+2}(\mathscr{F} ; A \oplus A) \rightarrow L_{2 k+2}\left(\mathbf{Z}\left[\mathbf{Z}_{m}\right]\right)
$$

where the ring epimorphism $\mathscr{F}: \mathbf{Z}[\mathbf{Z}] \rightarrow \mathbf{Z}\left[\mathbf{Z}_{m}\right]$ is induced by the inclusion map $\mathrm{Cl}(M-R(J)) \rightarrow M$ corresponds to the geometric process of glueing in $R(J)$. Needless, to say, the discussion here will rely upon notions and ideas set forth in $[\mathbf{S z}, \S 1]$. While we do not intend to reiterate the technical contents of that paper, we will include here helpful descriptions of terms and refer the reader to the above paper for more precise definitions.

We begin by recalling the definition of $\mathrm{b}_{2 n}(\mathscr{F} ; A \oplus B)$. Briefly, an element of this group is represented by

$$
\alpha=(\lambda: H \rightarrow G,(G ; \theta, \psi))
$$

where $\lambda$ is a homomorphism of $\mathbf{Z} \pi$ modules which induces an exact sequence $H_{\Lambda} \rightarrow G_{\Lambda} \rightarrow A \rightarrow 0$ after taking tensor product with $\Lambda$, and $(G ; \theta, \psi)$ is an $(-1)^{n}$-Hermitian form over $\mathbf{Z} \pi$ which determines a unimodular form over $\Lambda$ after taking tensor product with $\Lambda$. There are some further properties satisfied by the form and the homomorphism which will be discussed as needed. We begin with this description of $\alpha$ as a form on $H$ which factors through a form on $G$ with the property that the factorization is unimodular after tensoring with the "weaker" ring $\Lambda$. Note that the unimodular form $\left(G_{\Lambda} ; \theta_{\Lambda}, \psi_{\Lambda}\right)$ represents an element in $L_{2 n}(\Lambda)$.

We will show that this "forgetful" assignment induces a well-defined homomorphism $1_{*}: \mathbf{5}_{2 n}(\mathscr{F} ; A \oplus B) \rightarrow L_{2 n}(\Lambda)$. Since this assignment clearly respects the addition operations on forms and the obstruction groups are the equivalence classes of the respective additive relations, it suffices to show that the induced map on the groups is well-defined, i.e., if $\alpha$ and $\beta$ are two representatives of an element $\sigma \in \mathbf{b}_{2 n}(\mathscr{F} ; A \oplus B)$, then the corresponding unimodular forms are two representatives of $1_{*} \in$ $L_{2 n}(\Lambda)$; it is enough to check this for the trivial element. 
LemMA 2.1. Let $\alpha=(\lambda: H \rightarrow G,(G ; \theta, \psi))$ represent the trivial element of $\mathbf{b}_{2 n}(\mathscr{F} ; A \oplus B)$. Then, the associated unimodular form over $\Lambda$, $\left(G_{\Lambda} ; \theta_{\Lambda}, \psi_{\Lambda}\right)$ represents the trivial element of $L_{2 n}(\Lambda)$. Moreover, $(G ; \theta, \psi)$ represents the trivial element of $\Gamma_{2 n}(\mathscr{F})$.

Proof. By definition, $\alpha$ is equivalent to a representative $\beta$ which is strongly equivalent to zero (see [Sz, §1, p. 142]). That is, certain algebraic conditions are satisfied by the form and homomorphism of $\beta$. Essentially, these conditions amount to the existence of a submodule on which the form vanishes and a "splitting" of the form and homomorphism (over $\Lambda$ ) with respect to this submodule.

By [Sz, Lemma 1.4], we may choose $\beta$ to be of a very special type. There exist forms $\delta_{i}$ strongly equivalent to zero over $\mathrm{id}_{\mathbf{Z}_{\pi}}$ such that $\alpha \oplus \delta_{1} \oplus \delta_{2} \oplus \cdots \oplus \delta_{k}$ is strongly equivalent to zero. The operation, $\oplus$, as defined in [Sz, §1], is not simply orthogonal sum of forms. However, the effect of adding these $\delta_{i}$ 's is to change the unimodular form of $\alpha$ by a kernel in the sense of Wall. That is $\alpha$ is changed by something which is strongly equivalent to zero in the theory of Wall. It follows that we may as well assume that $\alpha$ is strongly equivalent to zero (in the sense of $\mathbf{b}$-theory) to begin with.

We will show that $\left(G_{\Lambda} ; \theta_{\Lambda}, \psi_{\Lambda}\right)$ is a Wall kernel [Wall, p. 47]. Let $K \subseteq H$ be a trivialization for $\alpha$, that is, on this submodule $K$, the induced from $(H ; \phi, \mu)$ vanishes and $K_{\Lambda}$ is a free direct summand of $H_{\Lambda}$ such that the composition
(*)
$\operatorname{coker} A \phi_{\Lambda \mid K_{\Lambda}} \rightarrow \operatorname{coker} A \phi_{\Lambda} \stackrel{\pi_{2}}{\rightarrow} B$

is an isomorphism, where $A \phi_{\Lambda}$ is the adjoint homomorphism of the form $\phi_{\Lambda}$, and coker $A \phi_{\Lambda} \cong A \oplus B$. Since $\alpha$ is strongly equivalent to zero, we may extend a basis for $K_{\Lambda}$ to one for $H_{\Lambda}=K_{\Lambda} \oplus Q_{\Lambda}$ so that

$$
A \phi_{\Lambda}=\left(\begin{array}{l|l}
0 & \delta \\
\hline \delta^{*} & 0
\end{array}\right)
$$

in terms of this decomposition. The restriction to $Q_{\Lambda}$ yields the exact sequence

$$
0 \rightarrow B^{*} \rightarrow Q_{\Lambda} \rightarrow K_{\Lambda}^{*} \rightarrow A \rightarrow 0,
$$

where the identification $A=\operatorname{coker} \delta$ is derived from (*) and in a similar manner $B^{*}=\operatorname{ker} \delta$. We have the following commutative diagram, over $\Lambda$,

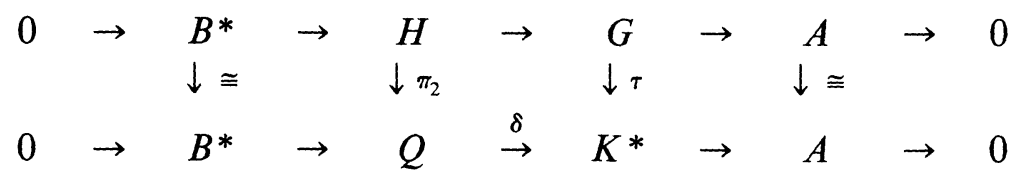

where $\tau=\pi_{1} \cdot \lambda^{*} \cdot A \theta: G \rightarrow K^{*} \subseteq H^{*}$. 
That the last vertical map is an isomorphism follows from the definition of a factorization $\lambda$ and a trivialization $K$; in particular, from the identification of the two cokernels with $A \subset A \oplus B \cong \operatorname{coker} A \phi_{\Lambda}$. Similarly, taking advantage of the duality required of a representative $\alpha$, that is,

$$
\operatorname{ker} A \phi_{\Lambda} \cong\left(\operatorname{coker} A \phi_{\Lambda}\right)^{*}
$$

[Sz, p. 7], we see that the first vertical map is an isomorphism. From this diagram we may conclude that $K_{\Lambda}^{*}$ is a direct summand of $G_{\Lambda}$ and that $\lambda \mid Q_{\Lambda}=\delta$ and $\lambda \mid K_{\Lambda}$ is an isomorphism onto $\operatorname{ker} \tau$. We obtain a splitting of $\tau$ by lifting a basis of the direct summand $K_{\Lambda}^{*} \subset H_{\Lambda}$ to $G_{\Lambda}$. This basis for $K_{\Lambda}^{*} \subset G_{\Lambda}$ may be extended to a basis of $G_{\Lambda}$ so that $\lambda$ is the direct sum of the restrictions $\lambda \mid K_{\Lambda}$ and $\lambda \mid Q_{\Lambda}$. It readily follows from the factorization of the adjoint map

$$
A \phi_{\Lambda}=\lambda^{*} \cdot A \theta_{\Lambda} \cdot \lambda
$$

that in terms of this basis,

$$
A \theta_{\Lambda}=\left(\begin{array}{c|c}
0 & I \\
\hline \pm I & \gamma
\end{array}\right)
$$

We may even arrange that $\gamma=0$ by choosing the splitting of $\tau: G_{\Lambda} \rightarrow K_{\Lambda}^{*}$ more carefully. Hence, $\left(G_{\Lambda} ; \theta_{\Lambda}, \psi_{\Lambda}\right)$ represents zero in the Wall group.

The last statement of the Lemma follows from a generalization of the above argument using the fact that the image of the trivialization of $\alpha$, $\lambda(K)$ is a pre-subkernel for $(G ; \theta, \psi)$ in the sense of Cappell-Shaneson [CS, p. 286] and so, $(G ; \theta, \psi)$ represents zero in the homology surgery group $\Gamma_{2 n}(\mathscr{F})$.

The next thing we will discuss is the geometic realization of the two forgetful homomorphisms

$$
1_{*}: \mathbf{b}_{2 n}(\mathscr{F} ; A \oplus B) \rightarrow L_{2 n}(\Lambda)
$$

and,

$$
1_{*}: \mathbf{b}_{2 n}(\mathscr{F} ; A \oplus B) \rightarrow \Gamma_{2 n}(\mathscr{F}) .
$$

To realize the first of these, we consider the following situation. Let $f:(P, \partial P) \rightarrow(Y, \partial Y)$ be maps of pairs such that $f|\partial P=g| \partial P$ and $h=f \cup g$ is a homotopy equivalence,

$$
\operatorname{dim}(X \cup Y)=\operatorname{dim}(\partial Y)+1=2 n-1
$$


We have the following diagrams

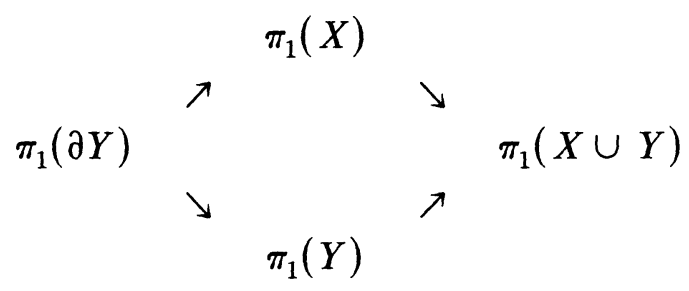

and for all $i$,

$$
\begin{array}{cccccc} 
& & K_{i}(P ; \Lambda) & & \\
& \nearrow & & \searrow & \\
& \searrow & & & & \\
& & K_{i}(\partial P ; \Lambda) & & & \\
& & & & & \\
& & & &
\end{array}
$$

where $\Lambda=\mathbf{Z}\left[\pi_{1}(X \cup Y)\right]=\mathbf{Z}[\pi]$. Hence,

$$
K_{i}(\partial P ; \Lambda) \cong K_{i}(P ; \Lambda) \oplus K_{i}(Q ; \Lambda)
$$

for all $i$. Let

$$
\begin{gathered}
\mathscr{F}: \mathbf{Z}\left[\pi_{1} Y\right] \rightarrow \Lambda, \quad A=K_{n-1}(P ; \Lambda) \quad \text { and } \\
B=K_{n-1}(P, \partial P ; \Lambda) \cong K_{n-2}(Q ; \Lambda) .
\end{gathered}
$$

By [Sz, Theorem 1.11], each element $\gamma \in \mathbf{b}_{2 n}(\mathscr{F} ; A \oplus B)$ may be realized as the surgery obstruction of a highly connected normal cobordism $(F, B): W \rightarrow Y \times I$ from $(f, b)$, relative the boundary, to a map $f_{0}:\left(P_{0}, \partial P\right) \rightarrow(Y, \partial Y)$ with the same properties over $\Lambda$. That is, the above diagrams are unchanged if we replace $P$ by $P_{0}$. It follows that $h_{0}=$ $f_{0} \cup g$ is a homotopy equivalence.

\section{THEOREM 2.2. There is a well-defined homomorphism}

$$
1_{*}: \mathrm{b}_{2 n}(\mathscr{F} ; A \oplus B) \rightarrow L_{2 n}(\pi) \text {. }
$$

Moreover, $1_{*}$ may be realized geometrically, i.e., in the situation described above, $1_{*}(\gamma)=\sigma((g \times \mathrm{id}) \cup F)$.

Proof. The first statement follows from Lemma 2.1, and the discussion preceding it. The second requires further discussion.

To show that $1_{*}(\gamma)=\sigma((g \times$ id $\cup F)$, we consider first the case when the generators of $A=K_{n-1}(P ; \Lambda)$ are spherical. Suppose that there exists a set of imbedded spheres in $\partial P$ whose images in $P$ generate $K_{n-1}(P ; \Lambda)$. If we assume that $f \mid \partial P$ is highly connected, this condition is clearly satisfied. Since these are imbedded in $\partial P$ we may arrange that 
the images in $P$ are disjoint and unlinked by viewing each in its own copy of $\partial P \times I$ and applying the arguments of $[\mathbf{S z}, 1.2]$. We then use these spheres as in $[\mathbf{S z}, 1.2]$ to realize the unimodular form through which the form on $K_{n}(W ; \Lambda)$ factors and thus obtain an element $\sigma(F, B) \in$ $\mathbf{b}_{2 n}(\mathscr{F} ; A \oplus B)$ represented by

$$
\alpha=\left(\iota: K_{n}(W) \rightarrow K_{n}(W, P),\left(K_{n}(W, P) ; \theta, \psi\right)\right)
$$

where $\theta$ and $\psi$ are given respectively by the intersection and self-intersection numbers. Each of $(n-1)$-spheres bounds a disk in $Q$ since $f \cup g$ is a homotopy equivalence. We may arrange that these are disjoint in $Q \times I$ by again viewing each in its own copy of $Q$ and each sphere in its own copy of $(\partial P \times I) \times I$. It is easy to see that the $k$-chains resulting from the union of a generator for $K_{h}(W, P ; \Lambda)$ and the corresponding disk in $Q$ represent generators of the form which represents $\sigma((g \times$ id $) \cup F) \in$ $L_{2 n}(\pi)$, and as we have arranged that the disks are disjoint and spheres unlinked, the intersection and self-intersection forms on $K_{n}(W \cup Q \times I ; \Lambda)$ are just those on $K_{n}(W, P ; \Lambda)$.

For the general case, we must find the disjoint unlinked spheres which will represent generators of $A \cong K_{n-1}(P ; \Lambda)$, and then proceed similarly to the above. To do this we generalize the argument used in [Sz, 1.2] for the case of toral generators. We may view $F$ as the union of two maps $G$ and $F^{\prime}$ to the product $Y \times I$, where $G: U \rightarrow Y \times[0,1 / 2]$ is a cobordism relative $\partial P$ from $P$ to $M$ obtained as follows. Let $\left\{x_{i}\right\}, i=1, m$ represent classes in $K_{n-1}(\partial P)$ whose images in $P$ generate $K_{n-1}(P ; \Lambda)$. These classes may be represented by cycles, each in its own copy of $\partial P \times I$. We perform surgeries on each copy so that the resulting map is highly connected. That is, the map restricted to $\partial P \times I$ is normally cobordant, via a highly connected map, $g: Z \rightarrow \partial P \times I \times I$, to a highly connected map. We replace $F: W \rightarrow Y \times I$ by a map represented schematically by

\begin{tabular}{|c|c|c|c|}
\hline$F$ & \multicolumn{3}{|c|}{$f \mid \partial P \times$ id $\times$ id } \\
\hline \multirow{2}{*}{$f \times$ id } & $-g^{\prime}$ & $-g^{\prime}$ & $-g^{\prime}$ \\
\cline { 2 - 4 } & $g^{\prime}$ & $g^{\prime}$ & $g^{\prime}$ \\
\hline
\end{tabular}

and now $G: U \rightarrow Y \times[0 \times 1 / 2]$, where $U$ is the union of the support of the surgeries in each copy of $\partial P \times I$. Now generators of $K_{n-1}(P ; \Lambda)$ are represented by spheres in $M$ which we have arranged to be disjoint and unlinked. We may proceed as above. We do note that although the surgeries may change the form $\alpha$,

$$
\sigma(F, B)=\sigma\left(F^{\prime} \cup G\right) \in \mathbf{b}_{2 n}(\mathscr{F} ; A \oplus B) .
$$


In fact, the effect of taking the union with $g^{\prime} \cup-g^{\prime}$ is to add a form trivial over $\mathbf{Z}[\pi]$, thus changing the unimodular form by a Wall kernel.

3. We now turn our attention to the comparison of $C(\rho, \tau)$ and $C\left(\rho, \tau_{0}\right)$ where $\tau$ is the restriction of $\rho$ to an invariant highly connected manifold $K$ and $\tau_{0}$ is the restriction to an invariant sphere.

While not every free $\mathbf{Z}_{m}$ action on $S^{2 k+1}$ has an invariant codimension two sphere, this is the case if $k$ and $m$ are not both even, [CS, Corollary 9.3].Further, the obstruction defined in [CS, 9.2] to a particular $\tau_{0}$ arising as the restriction of $\rho$ to an invariant sphere lies in $\mathbf{Z}_{2}$. Thus, it is very often the case, that given $\tau=\rho \mid K$, we may produce a $\tau_{0}=\rho \mid \Sigma$, a restriction to a homotopy sphere.

Let $J=K / \tau$ be a highly connected characteristic submanifold [see $\S 1]$ of the homotopy lens space $M=S^{2 k+1} / \rho$ with characteristic map $f: M \rightarrow N$, restricting to a map $f \mid J: J \rightarrow L$ where $N$ is a suspension of a homotopy lens space $L$. We will assume that what is most likely is in fact true, i.e., $L=\Sigma / \tau_{0}$ is a characteristic submanifold of $M, \tau_{0}=\rho \mid \Sigma$.

In [CS, 10.3], a classification of $C\left(\rho, \tau_{0}\right)$ is obtained in terms of homology surgery groups. In particular, it is shown that there is a subgroup of the cokernel of the natural map

$$
L_{2 k+2}(\mathbf{Z}) \rightarrow \Gamma_{2 k+2}(\mathscr{F})
$$

which acts freely on $C\left(\rho, \tau_{0}\right)$ with orbit space $0, \mathbf{Z}_{2}$, or $\mathbf{Z}$ depending upon the parities of $k$ and $m$.

By our classification result, Theorem 1.5, for $\pi_{k-1}(J)=0$, there exists a subgroup $B_{0} \subseteq \mathbf{b}_{2 k+2}(\mathscr{F} ; 0 \oplus 0)=\Gamma_{2 k+2}(\mathscr{F})$ such that $\tilde{B}_{0}$ acts freely on $C\left(\rho, \tau_{0}\right)$ where $\tilde{B}_{0}$ is the quotient of $B_{0}$ by the image of the composite of the surgery obstruction map

$$
\left[\Sigma^{2} S^{2 k-1} \times S^{1}, G / \mathrm{PL}\right] \rightarrow L_{2 k+2}(\mathbf{Z})
$$

and the natural map $L_{2 k+2}(\mathbf{Z}) \rightarrow \Gamma_{2 k+2}(\mathscr{F})$. This constitutes an alternate description of the subgroup and action described in [CS, Corollary 10.4].

This description has the advantage of pointing towards a group structure on $C\left(\rho, \tau_{0}\right)$. Let $\xi$ be the normal bundle of the embedding $L \subset M$ with total space $E(\xi)$. In this case, every embedding $L \subset M$ factors through a semi-local knot into $E(\xi)$ over the identity map id ${ }_{L}$. The cobordism classes of such semi-local knots is denoted by $C\left(\mathrm{id}_{L}, \xi\right)$, (see [Sz, p. 153]). Combining this with Proposition 1.3, we may identify $C\left(\rho, \tau_{0}\right)$ with $C\left(\mathrm{id}_{L}, \xi\right)$ in [Sz, p. 153]. There is a group operation defined on the latter set. Let $\iota_{1}$ and $\iota_{2}$ be two semi-local knots, that is, embeddings of $L$ into $E(\xi)$. The composition or tunnel sum of $\iota_{1}$ and $\iota_{2}$, written 
$\iota_{1} \cdot \iota_{2}$, is defined in [CS, p. 305] as follows. Let $T$ be a tubular neighborhood of $\iota_{1}(L)$ and let $g: T \rightarrow E(\xi)$ be the canonical bundle map. Then, $\iota_{1} \cdot \iota_{2}$ is defined to be the composition.

$$
L \stackrel{\iota_{2}}{\rightarrow} E(\xi) \stackrel{g^{-1}}{\rightarrow} T \subseteq E(\xi) .
$$

This is well-defined, up to isotopy.

It follows as above that $C(\rho, \tau)$ may be identified with $C(f \mid J, \xi)$, the set of cobordism classes of semi-local knots over $f \mid J$ into $\xi$. We define $\mathscr{B}$, an action of $C\left(\rho, \tau_{0}\right)$ on $C(\rho, \tau)$ by tunnel sum. Let $\iota_{1}$ and $\iota_{2}$ represent elements of $C\left(\mathrm{id}_{L}, \xi\right)$ and $C(f \mid J, \xi)$ respectively. The action of $\iota_{1}$ on $\iota_{2}$ is defined to be the composition

$$
J \stackrel{\iota_{2}}{\rightarrow} E(\xi) \stackrel{g^{-1}}{\rightarrow} T \subseteq E(\xi)
$$

where $g$ and $T$ are as above.

As we saw in the previous section, there is a well-defined action of $\Gamma_{2 k+2}(\mathscr{F})$ on $\mathbf{b}_{2 k+2}(\mathscr{F} ; A \oplus A)$ given by orthogonal sum of forms. Let $\tilde{B}_{0}$ and $\tilde{B}$ be the groups defined in Theorem 1.5 for $C\left(\rho, \tau_{0}\right)$ and $C(\rho, \tau)$ respectively. Consider the diagram

$$
\begin{gathered}
\Gamma_{2 k+2}(\mathscr{F}) \underset{j_{*} \searrow}{\stackrel{\mathscr{A}}{\rightarrow}} \mathbf{b}_{2 k+2}(\mathscr{F} ; A \oplus A) \\
L_{2 k+2}\left(\mathbf{Z}_{m}\right)
\end{gathered}
$$

where $\mathscr{A}$ is given by the action mentioned above and the homomorphisms to $L_{2 k+2}\left(\mathbf{Z}_{m}\right)$ are the "forgetful" homomorphisms. It is obvious from the definitions that the diagram commutes. It follows that $\mathscr{A}$ restricts to $\mathscr{A} \mid B_{0}: B_{0} \rightarrow B$. Similarly, the diagram

$$
\mathbf{b}_{2 k+2}(\mathscr{F} ; 0)=\Gamma_{2 k+2}(\mathscr{F}) \stackrel{\mathscr{A}}{\rightarrow} \mathbf{b}_{2 k+2}(\mathscr{F} ; A \oplus A)
$$

commutes where the homomorphisms from $L_{2 k+2}(\mathbf{Z})$ are given by the orthogonal sum action on the $\mathbf{b}$-surgery groups; it follows that there is a well-defined homomorphism

$$
\mathscr{A}: \tilde{B}_{0} \rightarrow \tilde{B} \text {. }
$$

To appreciate the relationship between the algebraically defined action $\mathscr{A}$ given by orthogonal sum and the geometrically defined action $\mathscr{B}$ given by tunnel sum, we consider the geometric interpretation of $\mathscr{A}$ given by the realization theorem of surgery theory. 
THEOREM 3.1. There exists a homomorphism $\mathscr{A}: \tilde{B}_{0} \rightarrow \tilde{B}$ and an action $\mathscr{B}: C\left(\rho, \tau_{0}\right) \rightarrow C(\rho, \tau)$ such that the diagram

$$
\begin{aligned}
0 \rightarrow & \tilde{B}_{0} \rightarrow C\left(\rho, \tau_{0}\right) \\
& \downarrow \mathscr{A} \quad \downarrow \mathscr{B} \\
0 \rightarrow & \tilde{B} \rightarrow C(\rho, \tau)
\end{aligned}
$$

commutes.

Proof. Let $\mathscr{A}$ and $\mathscr{B}$ be as described above. Let $\gamma$ be an element of $B_{0} \subset \Gamma_{2 k+2}(\mathscr{F})=\mathbf{b}_{2 k+2}(\mathscr{F} ; 0), x \in C(\rho, \tau)$. We may view $x$ as a semilocal knot into a 2-disk bundle $\xi$ over $f \mid J: J \rightarrow L$. Let $h_{x}=f \mid W_{x}$ be the restriction of $f$ to the complement of the semi-local knot representing $x$, where $f$ is the characteristic map. We may realize $\gamma \in B_{0}$ by a cobordism, $G$, relative boundary from the identity on $S(\xi) \times I$ to the complementary map for a semi-local knot over id: $L \rightarrow L$. Let $H=h_{x} \times$ id; taking the union along the identity on $S(\xi) \times 0 \times I$, we see that $\mathscr{A}(\gamma)=\sigma(G \cup H)$; also, we have that $G \cup H \cup\left(f \mid T(J) \times \mathrm{id}_{I}\right)$ is a cobordism between the characteristic maps of the semi-local knots representing $x$ and $\mathscr{A}(\gamma) \cdot x$. But this cobordism is exactly the same as the one obtained from the description of the tunnel sum operation of $\gamma \cdot 0 \in C\left(\rho, \tau_{0}\right)$ on $x \in$ $C(\rho, \tau)$.

Next we investigate the relationship between $O\left(\tau_{0}\right)$ and $O(\tau)$, the orbit spaces of the actions of $B_{0}$ and $B$, respectively. Consider $C\left(\rho, \tau_{0}\right)$ as the classes of semi-local knots of $L \subset E(\xi), \partial E=S(\xi)=S^{2 k-1} \times S^{1}$. Recall that two elements $x$ and $y$ in $C\left(\rho, \tau_{0}\right)$ will be in the same orbit if and only if there is a cobordism $F$ between their characteristic maps, $f_{x}$ and $f_{y}$, such that $V=F^{-1}(S(\xi) \times I)$ is an $s$-cobordism and $F \mid V$ is a simple homotopy equivalence; in particular, $V$ is a diffeomorphic to $S(\xi) \times I$. For the case at hand, we note that by Lemma 1.3 and the definition of $C\left(\rho, \tau_{0}\right)$ there is a normal cobordism, $G$, from $f_{x}$ to $f_{y}$ with underlying manifold an s-cobordism. Viewing $x$ and $y$ as semi-local knots and arguing as in [Sz, 2.5 and 2.6], we conclude that $x$ and $y$ are in the same orbit if and only if this map $G$ is normally cobordant relative $f_{x} \cup f_{y}$ to a map $F$ with the properties described. The obstruction to finding such a map is just the surgery obstruction $\sigma\left(G \mid G^{-1}(S(\xi) \times I)\right) \in L_{n+2}(\mathbf{Z})$. By [Sh, 5.1], $L_{n+2}(\mathbf{Z}) \cong L_{n+1}(e)$. For $C(\rho, \tau)$, we view $x$ and $y$ as semi-local knots into $E(\xi)$ over $g: J \rightarrow L$. Recalling that the normal invariant of $g$ necessarily vanishes and arguing as in [Sz, Lemma 2.5], we may apply the same reasoning to this case to conclude that $O(\tau)$ is given in a similar fashion as the surgery obstruction of the restriction (to the appropriate 
subspace) of a normal cobordism between the characteristic maps $f_{x}$ and $f_{y}$. The vanishing of this obstruction implies that the restriction is normally cobordant relative boundary to a map of $s$-cobordisms [Sz, Lemma 2.6].

THEOREM 3.2. The natural map $\overline{\mathscr{B}}: O\left(\tau_{0}\right) \rightarrow O(\tau)$, induced by the operation of tunnel sum, is a bijection.

Proof. That $\overline{\mathscr{B}}$ is one to one follows easily from the definition of tunnel sum and the action of $B_{0}$ on $B$. Again, we view elements of $C(\rho, \tau)$ as semi-local knots over $g: J \rightarrow L$. Since $g$ is normally cobordant to $\mathrm{id}_{L}$, the cobordism $G \mid G^{-1}(S(\xi) \times I)$ described above determines an element $\sigma$ in the image of

$$
[\Sigma L, G / \mathrm{PL}] \stackrel{s_{h}}{\rightarrow} L_{n+1}\left(\mathbf{Z}_{m}\right) \stackrel{p^{!}}{\rightarrow} L_{n+2}(\mathbf{Z}),
$$

$p^{!}$induced by the projection $p: S(\xi) \rightarrow L$. By the argument in [CS, pp. $331-332]$, the image of this composition is $O\left(\tau_{0}\right)$. Hence, $\overline{\mathscr{B}}$ is onto.

We conclude with a description of the orbit space of the action of $\tilde{B}$ on $C(\rho, \tau)$.

THEOREM 3.3. The orbit space, $O(\tau)$, (where $n=2 k-1)$ is given by

$$
O(\tau)= \begin{cases}0, & k \text { odd }, \text { m even } ; \\ \mathbf{Z}_{2}, & \dot{k} \text { odd }, \text { m odd } ; \\ \mathbf{Z}, & k \text { even } .\end{cases}
$$

Proof. This is a direct consequence of the above theorem and [CS, Corollary 10.4].

4. Combining Theorems 3.1 and 3.2 , we have the diagram of exact sequences

$$
\begin{aligned}
0 & \rightarrow \tilde{B}_{0} \rightarrow C\left(\rho, \tau_{0}\right) \rightarrow O\left(\tau_{0}\right) \rightarrow 0 \\
& \downarrow \tilde{\mathscr{A}} \quad \downarrow \mathscr{B} \quad \downarrow \\
0 & \rightarrow \tilde{B} \rightarrow C(\rho, \tau) \rightarrow O(\tau) \rightarrow 0
\end{aligned}
$$

where $\tilde{\mathscr{A}}$ is the homomorphism derived from the action of $\mathscr{A}$ of $\Gamma_{2 k+2}(\mathscr{F})$ on $\mathbf{b}_{2 k+2}(\mathscr{F} ; A \oplus A)$ and $\mathscr{B}$ is the action given by tunnel sum. Thus, any questions concerning the orbit space of the geometric action may be rephrased in purely algebraic terms. In this section, the quotient of 
$\mathrm{B}_{2 k+2}(\mathscr{F} ; A \oplus B)$ by the action of $\Gamma_{2 k+2}(\mathscr{F})$ is shown to depend upon $A \oplus B$. (In the geometric situation studied in the previous sections, $B \cong A$.) In particular, if $A^{*} \neq 0$, the orbit space of $\mathscr{A}$ is non-trivial and in fact quite large. Hence, it will follow that the collection of equivariant embeddings of a highly connected submanifold is significantly larger than the set of equivariant embeddings of a homotopy sphere in the case $A^{*} \neq 0$. If $A^{*}=0$, we have that $\mathscr{B}$ is a transitive action, or has an orbit space with at most two elements, depending in part upon whether the group $\mathbf{Z}_{m}$ is of odd or even order.

Let

$$
\alpha=(\lambda: H \rightarrow G,(G ; \theta, \psi))
$$

represent an element in $\mathbf{5}_{2 k+2}(\mathscr{F} ; A \oplus B)$ and $\beta=(G ; \theta, \psi)$ an element in $\Gamma_{2 k+2}(\mathscr{F})$. As a consequence of the proof of Lemma 2.1 we have the following statement concerning the notions of strongly equivalent to zero in the two theories (see [Sz, p. 142] and [CS, p. 286]).

LEMMA 4.1. If $\alpha$ is strongly equivalent to zero in the sense of Б-surgery theory, then $\beta$ is strongly equivalent to zero in the sense of $\Gamma$-surgery theory.

An analogous statement holds for notions of "equivalent to zero" in the respective surgery groups. The converse is not true, in general, and it is this that is closely related to the question concerning the orbit space of the action $\mathscr{A}$.

Using the above lemma, it is easy to formulate an alternative definition for the notion of "strongly equivalent to zero" which we state here without verification. Recall that the form on $G$ induces a form on $H$. The kernel of the adjoint of the form on $H_{\Lambda}$ is isomorphic to the dual of the cokernel of this adjoint, and so, it may be identified as $A^{*} \oplus B^{*}$.

Proposition 4.2. The form $\alpha$ is strongly equivalent to zero if and only if $\lambda(H) \subseteq G$ contains a pre-subkernel $K$ (as defined in [CS, pp. 286-7]) such that over the coefficient ring $\Lambda, \lambda \mid A^{*}$ maps $A^{*}$ monomorphically onto a submodule of $K_{\Lambda}$.

Thus, the orbit space will involve the submodules $\lambda(H)$ but not their extensions by $B^{*}$. In the discussions and proofs to follow, it is useful to think in terms of the case $B^{*}=0$.

THEOREM 4.3. If $A^{*} \neq 0$, the action of $\Gamma_{2 k+2}(\mathscr{F})$ on

$$
\mathbf{b}_{2 k+2}(\mathscr{F} ; A \oplus B)
$$

is not transitive. 
Proof. It suffices to show that there exist non-zero elements of $\mathbf{b}_{2 k+2}(\mathscr{F} ; A \oplus B)$ with representatives

$$
\alpha=(\lambda: H \rightarrow G,(G ; \theta, \psi))
$$

such that $\beta=(G ; \theta, \psi)$ represents zero in $\Gamma_{2 k+2}(\mathscr{F})$. This follows from the fact that the orbit space may be identified with the kernel of the "forgetful" map

$$
1_{*}: \mathbf{b}_{2 k+2}(\mathscr{F} ; A \oplus B) \rightarrow \Gamma_{2 k+2}(\mathscr{F}) .
$$

Let $\beta$ be a fixed representative of zero in $\Gamma_{2 k+2}(\mathscr{F})$; we may assume that $\beta$ is strongly equivalent to zero. For any homomorphism $\lambda: H \rightarrow G$ of free $\mathbf{Z}[\pi]$-modules with coker $\left(\lambda \oplus_{\mathbf{z}_{\pi}} \mathrm{id}_{\Lambda}\right)=A$ and $\operatorname{ker}\left(\lambda \oplus_{\mathbf{z}_{\pi}} \mathrm{id}_{\Lambda}\right)=$ $B^{*}$, the induced form $\alpha$ will be strongly equivalent to zero only if the form on $G$ vanishes on $\lambda\left(A^{*}\right) \subset G$. By composing with an automorphism of $H$, we may easily arrange the failure of this condition. Clearly, stabilization does not alter the form on the submodule $\lambda\left(A^{*}\right)$ and so can not correct for this failure.

By first considering the situation $\Lambda=\mathbf{Z}$, we may deduce that this orbit space is in fact quite large when $A^{*} \neq 0$ and $\Lambda=\mathbf{Z}[\pi]$, for $\pi$ a finite group.

Let $\mathscr{F}_{0}: \mathbf{Z}[\pi] \rightarrow \mathbf{Z}$ be an epimorphism and write $A$ as a direct sum of its free and torsion parts, $A=F(A) \oplus \operatorname{Tor}(A)$. Once more taking advantage of properties of the principal ideal domain $\mathbf{Z}$, we observe that $A^{*}$ will be a direct summand of both $H$ and $G$ over the coefficients and we may actually write

$$
\alpha=\left(\lambda: A^{*} \oplus F=H \rightarrow G=A^{*} \oplus F(A) \oplus E,(G ; \theta, \psi)\right) .
$$

We make the following observations concerning the restrictions of $\lambda$ and the adjoint map, $A \theta$ over $\Lambda$ with respect to this decomposition of $G$ and $H$.

(1) $\lambda \mid A^{*}$ is an isomorphism onto $A^{*}$;

(2) $A \theta \mid A^{*}$ is an isomorphism onto $F(A)^{*} \subset G^{*}$ and $F(A)^{*}$ is identified with $A^{*}=\operatorname{ker} \lambda^{*}$;

(3) $A \theta \mid F(A)$ is an isomorphism onto $A^{* *} \subseteq G^{*}$ which in turn, by the statement dual to (1) and the restriction of the composition

$$
G^{*} \rightarrow H^{*} \rightarrow A \oplus B \rightarrow 0
$$

is identified with $F(A) \subset A \oplus B$;

(4) the restriction of $\theta$ to $A^{*} \oplus F(A)$ is a Wall kernel. It follows that $\alpha$ determines, by restriction, a form over $\Lambda$,

$$
\alpha \mid=(\lambda \mid F: F \rightarrow E,(E ; \theta, \psi))
$$


which represents an element in $\mathbf{b}_{2 k+2}\left(\mathrm{id}_{\mathbf{z}} ; \operatorname{Tor}(A) \oplus B\right)$. We may argue from the definitions that this determines a map of the surgery groups

$$
r_{*}: \mathbf{b}_{2 k+2}\left(\mathrm{id}_{\mathrm{z}} ; A \oplus B\right) \rightarrow \mathbf{b}_{2 k+2}\left(\mathrm{id}_{\mathrm{z}} ; \operatorname{Tor}(A \oplus B)\right) \text {. }
$$

Using the reciprocity formula which we discuss below, we identify the target group with the Wall group $L_{2 k+2}(e)$. The homomorphism

$$
\mathbf{b}_{2 k+2}\left(\mathscr{F}_{0} ; A \oplus B\right) \rightarrow L_{2 k+2}(e)
$$

is the same as the composition of the natural surjections

$$
\mathbf{b}_{2 k+2}\left(\mathscr{F}_{0} ; A \oplus B\right) \stackrel{1_{*}}{\rightarrow} \Gamma_{2 k+2}\left(\mathscr{F}_{0}\right) \stackrel{j_{*}}{\rightarrow} L_{2 k+2}(e) .
$$

For the case $A^{*}=0$, we have

$$
\mathbf{b}_{2 k+2}\left(\mathscr{F}_{0} ; \operatorname{tor}(A \oplus B)\right) \stackrel{1_{*}}{\rightarrow} \Gamma_{2 k+2}\left(\mathscr{F}_{0}\right) \stackrel{j_{*}}{\rightarrow} L_{2 k+2} ;
$$

It follows from the reciprocity formula that the map $1_{*}$ is an isomorphism. We are interested in the comparative sizes of the kernels of the two compositions. We may construct a monomorphism

$$
k_{*}: \mathbf{b}_{2 k+2}\left(\mathscr{F}_{0} ; \operatorname{tor}(A \oplus B)\right) \rightarrow \mathbf{b}_{2 k+2}\left(\mathscr{F}_{0} ; A \oplus B\right)
$$

as follows. Let $K$ be a free $\mathbf{Z}[\pi]$ module of the same rank as the free abelian group $A^{*}$. Let $\kappa$ be the standard kernel over $\mathbf{Z}[\pi]$ with free module $K \oplus K^{*}$, that is, $\kappa$ is the canonical representative of the trivial element in both $L_{2 k+2}(\pi)$ and $\Gamma_{2 k+2}\left(\mathscr{F}_{0} ; \mathbf{Z}[\pi] \rightarrow \mathbf{Z}\right)$. Suppose

$$
\alpha_{0}=(\lambda: H \rightarrow G,(G ; \theta, \psi))
$$

represents an element in $\mathbf{b}_{2 k+2}\left(\mathscr{F}_{0} ; \operatorname{tor}(A \oplus B)\right)$. Then $k_{*}\left(\alpha_{0}\right)$ is represented by

$$
\alpha=\left(\lambda \perp \iota: H \oplus K \rightarrow G \oplus K \oplus K^{*},((G ; \theta, \psi) \perp \kappa)\right),
$$

where $\iota$ is the inclusion onto the first factor of $K \oplus K^{*}$. We note that $K_{\Lambda}$ is identified with the direct summand $A^{*}$ of the kernel of the form on $(H \oplus K)_{\Lambda}$. Checking the definitions, we readily see that this is well-defined. Moreover, this same construction produces a homomorphism

$$
k_{*}: \mathbf{b}_{2 k+2}\left(\mathrm{id}_{\mathbf{z}} ; \operatorname{tor}(A \oplus B)\right) \rightarrow \mathbf{5}_{2 k+2}\left(\mathrm{id}_{\mathbf{z}} ; A \oplus B\right)
$$

which is an inverse to $r_{*}$.

Now to see that the kernel of

$$
\mathbf{b}_{2 k+2}\left(\mathscr{F}_{0} ; A \oplus B\right) \rightarrow \Gamma_{2 k+2}\left(\mathscr{F}_{0}\right)
$$

contains an infinite number of elements we mimic the construction of the homomorphism $k_{*}$. In this case, let $\alpha_{*}$ represent the trivial element of $\mathbf{b}_{2 k+2}\left(\mathscr{F}_{0} ; A \oplus B\right)$ and replace the trivial $\kappa$ by any form $\beta$ on $K \oplus K^{*}$ 
representing an element in the kernel of $j_{*}: \Gamma_{2 k+2}\left(\mathscr{F}_{0}\right) \rightarrow L_{2 k+2}(e)$. It is well known that this kernel is quite large. Combining $\alpha_{0}$ and $\beta$, as before, we obtain an element $\alpha$ representing an element in $\mathbf{b}_{2 k+2}\left(\mathscr{F}_{0} ; A \oplus B\right)$, not in the image of $k_{*}$ and in the kernel of $j_{*}$. That distinct choices of the equivalence classes $[\beta]$ determine distinct classes $[\alpha]$ follows since $1_{*}[\alpha]=$ $1_{*}[\beta]$. We summarize this discussion in the following.

Proposition 4.4. Let $\mathscr{F}_{0}: \mathbf{Z}[\pi] \rightarrow \mathbf{Z}$ be a ring epimorphism. The homomorphism

$$
1_{*}: \mathbf{b}_{2 k+2}\left(\mathscr{F}_{0} ; A \oplus B\right) \rightarrow \Gamma_{2 k+2}\left(\mathscr{F}_{0}\right),
$$

is an isomorphism is $A^{*}=0$. If $A^{*} \neq 0, \operatorname{ker} 1_{*}$ is infinite.

To reach our desired geometric conclusion, we combine Proposition 4.4 with the observation that the images of the natural maps

$$
\Gamma_{2 k+2}(\mathscr{F}) \rightarrow \Gamma_{2 k+2}\left(\mathscr{F}_{0}\right)
$$

and

$$
\mathbf{b}_{2 k+2}(\mathscr{F} ; A \oplus B) \rightarrow \mathbf{b}_{2 k+2}\left(\mathscr{F}_{0} ; A \oplus B\right)
$$

are large, $\mathscr{F}_{0}: \mathbf{Z}[\pi] \rightarrow \mathbf{Z}$ and $\mathscr{F}: \mathbf{Z}[\pi] \rightarrow \mathbf{Z}\left[\mathbf{Z}_{m}\right]$.

THEOREM 4.5. If $A^{*} \neq 0$, the action of $C\left(\rho, \tau_{0}\right)$ on $C(\rho, \tau)$ induced by tunnel sum is not transitive. The orbit space is not finite.

Proof. This is a restatement of Theorem 4.3 and a summary of the above discussion. The second statement follows from Proposition 4.4.

Thus, we see that in the case $A^{*} \neq 0$, there exists an infinite collection of equivariant embeddings of a given highly connected submanifold in $S^{2 k+1}$ which are not related by the operation of tunnel sum with an equivariant homotopy sphere.

To complete our study of the algebraic action, $\mathscr{A}$, we consider the case $A^{*}=0$. Let $\alpha=(\lambda: H \rightarrow G,(G ; \theta, \psi))$ represent an element in $\left(\operatorname{ker} 1_{*}\right) \subset \mathbf{b}_{2 k+2}(\mathscr{F} ; A \oplus B)$. We assume as before that $\beta=(G ; \theta, \psi)$ is strongly equivalent to zero. By Proposition 4.2 , in this case, $\alpha$ will be strongly equivalent to zero if and only if there exists a trivialization of $\beta$ which in fact is a trivialization of $\alpha$. That is, $\alpha$ is strongly equivalent to zero if and only if $\lambda(H)$ contains a pre-kernel. (Compare with Proposition 4.4). The set of stable equivalence classes of pre-subkernels of the 
trivial form in $\Gamma_{2 k+2}(\mathscr{F})$ is the surgery obstruction group $\Gamma_{2 k+1}(\mathscr{F}) \subset$ $L_{2 k+1}(\Lambda)$. In a future paper, we define $\mathbf{b}_{2 k+1}(\mathscr{F} ; A \oplus B)$ to be the subgroup of $\Gamma_{2 k+1}(\mathscr{F})$ whose elements have representatives which are trivializations of $\alpha$ as well as $\beta$. The relationship between the orbit space of the algebraic action, $\mathscr{A}$, and the quotient of these two groups is given by the following reciprocity formula.

THEOREM 4.6. Let $A^{*}=0$. There is an isomorphism between quotients of surgery obstruction groups which relates $\Gamma$-surgery theory to $\mathbf{6}$-surgery theory and odd dimensional groups to even dimensional groups. In particular, the natural map

$$
\mathbf{b}_{2 k+2}(\mathscr{F} ; A \oplus B) / \Gamma_{2 k+2}(\mathscr{F}) \rightarrow \Gamma_{2 k+3}(\mathscr{F}) / \mathbf{b}_{2 k+3}(\mathscr{F} ; A \oplus B)
$$

is an isomorphism.

For the geometric situation at hand, this theorem has the following consequence.

THEOREM 4.7. If $A^{*}=0$ and if either $k$ or $m$ is odd, the action of $C\left(\rho, \tau_{0}\right)$ on $C(\rho, \tau)$ is transitive; that is, any equivariant embedding of a highly connected submanifold of a $S^{2 k+1}$ is obtainable (up to cobordism) from any other by taking its tunnel sum with an equivariant embedding of a homotopy sphere. If both $k$ and $m$ are even, the orbit space has at most two elements.

Proof. By the above, the orbit space is given as the quotient of the inclusion

$$
\mathbf{b}_{2 k+3}\left(\mathscr{F}: \mathbf{Z}[\mathbf{Z}] \rightarrow \mathbf{Z}\left[\mathbf{Z}_{m}\right] ; A \oplus B\right) \rightarrow \Gamma_{2 k+2}\left(\mathscr{F}: \mathbf{Z}[\mathbf{Z}] \rightarrow \mathbf{Z}\left[\mathbf{Z}_{m}\right]\right) .
$$

But since $L_{2 k+3}\left(\mathbf{Z}_{m}\right)=0$ for $k$ or $m$ odd, this must be zero. The case of $k$ and $m$ even follows similarly since $L_{2 k+3}\left(\mathbf{Z}_{m}\right)=\mathbf{Z}_{2}$ in this case.

\section{REFERENCES}

[B] W. Browder, Surgery on Simply Connected Manifolds, Springer, 1972.

[CS] S. E. Cappell and J. L. Shaneson, The codimension two placement problem and homology equivalent manifolds, Annals of Math., 99 (1974), 277-348.

[M] J. Milnor, Whitehead torison, Bull. Amer. Math. Soc., 72 (1966), 358-426.

[Sh] J. L. Shaneson, Wall's surgery obstruction groups for $\mathbf{Z} \times G$, Annals of Math., 90 (1969), 296-334. 
[St] N. Stolzfus, Unraveling the integral knot concordance group, Memoirs Amer. Math. Soc., 192 (1977).

[Sz] S. Szczepanski, Highly connected embeddings in codimension two, Trans. Amer. Math. Soc., 280 (1983), 139-159.

[Sz2] _ Odd dimensional $\mathbf{5}$-surgery obstruction groups and the reciprocity principle, in preparation.

[W] C. T. C. Wall, Surgery on Compact Manifolds, Academic Press, 1970.

Received February 12, 1985.

LEHIGH UNIVERSITY

BETHLEHEM, PA 18016 


\section{PACIFIC JOURNAL OF MATHEMATICS EDITORS}

V. S. VARADARAJAN

(Managing Editor)

University of California

Los Angeles, CA 90024

Herbert Clemens

University of Utah

Salt Lake City, UT 84112

R. FINN

Stanford University

Stanford, CA 94305
ROBION KIRBY

University of California

Berkeley, CA 94720

C. C. MOORE

University of California

Berkeley, CA 94720

HAROLD STARK

University of California, San Diego La Jolla, CA 92093

\section{ASSOCIATE EDITORS}
R. ARENS
E. F. BECKENBACH
B. H. NEUMANN
F. WOLF
K. YosHIDA (1906-1982)

\section{SUPPORTING INSTITUTIONS}

UNIVERSITY OF ARIZONA

UNIVERSITY OF BRITISH COLUMBIA

CALIFORNIA INSTITUTE OF TECHNOLOGY

UNIVERSITY OF CALIFORNIA

MONTANA STATE UNIVERSITY

UNIVERSITY OF NEVADA, RENO

NEW MEXICO STATE UNIVERSITY

OREGON STATE UNIVERSITY
UNIVERSITY OF OREGON UNIVERSITY OF SOUTHERN CALIFORNIA

STANFORD UNIVERSITY

UNIVERSITY OF HAWAII

UNIVERSITY OF TOKYO

UNIVERSITY OF UTAH

WASHINGTON STATE UNIVERSITY

UNIVERSITY OF WASHINGTON 


\section{Pacific Journal of Mathematics}

Vol. 129, No. $1 \quad$ May, 1987

Aldridge Knight Bousfield, Uniqueness of infinite deloopings for

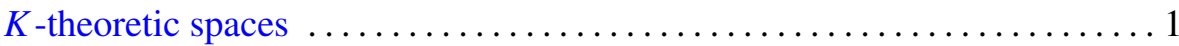

Mark Gregory Davidson, The harmonic representation of $U(p, q)$ and its

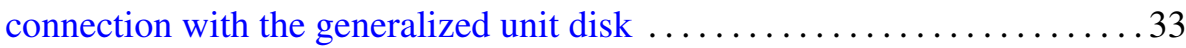

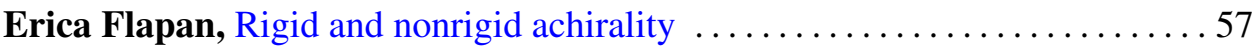

Peter Abraham Greenberg, Pseudogroups of $C^{1}$ piecewise projective

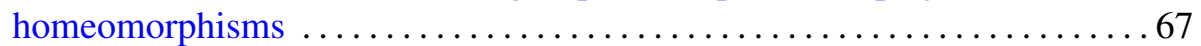

Peter Martin Knopf, Maximal functions on the unit $n$-sphere $\ldots \ldots \ldots \ldots 77$

Norman Jay Levitt and Andrew Ranicki, Intrinsic transversality

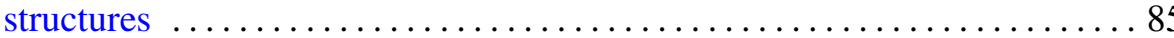

Susan Szczepanski, Invariant submanifolds of free cyclic actions on

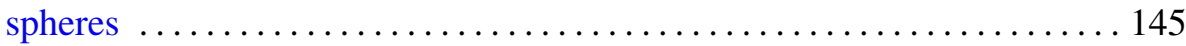

Kazimierz Szymiczek, Generalized rigid elements in fields . . . . . . . . 171

Domingo Toledo, Nonexistence of certain closed complex geodesics in the

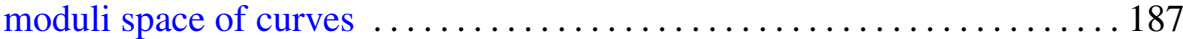

Graham H. Williams, The best modulus of continuity for solutions of the

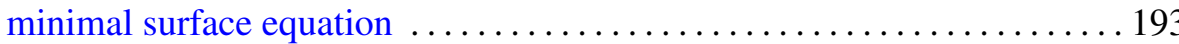

